\title{
A NONLINEAR MACROELEMENT FORMULATION FOR THE SEISMIC ANALYSIS OF MASONRY BUILDINGS
}

\author{
Domenico Liberatore $^{1}$, Daniela Addessi ${ }^{2}$ and Marialuigia Sangirardi ${ }^{2}$ \\ ${ }^{1}$ DISG, Faculty of Architecture, "Sapienza" University of Rome \\ Via Gramsci 53, 00197 Rome, Italy \\ e-mail: domenico.liberatore@uniroma1.it \\ ${ }^{2}$ DISG, Faculty of Engineering, "Sapienza" University of Rome \\ Via Eudossiana 18, 00184 Rome, Italy \\ e-mail: daniela.addessi@uniroma1.it \\ e-mail: marilusangirardi@gmail.com
}

Keywords: Masonry, Macroelement, Bouc-Wen-Baber-Noori.

\begin{abstract}
A macroelement is presented for the nonlinear dynamic analysis of masonry structures under seismic actions. The macroelement, developed in the framework of the equivalent frame model, has a force-based formulation and accounts for flexural and shear failure mechanisms, by means of two flexural hinges at the ends and a shear link, respectively. The flexural hinges are formulated according to the Bouc-Wen model to describe the progressive development of cracks and the hysteresis loops under load reversals. The shear link, in addition to the aforementioned effects, accounts for the strength/stiffness decay and is formulated adopting the Bouc-Wen-Baber-Noori model. Numerical comparisons with experimental tests on masonry piers are presented, showing the suitability of the presented macroelement.
\end{abstract}




\section{INTRODUCTION}

The in-plane response of masonry buildings under seismic actions is usually studied through pushover analysis, assuming that the response is dominated by a single mode. However, in the case of irregular plan/elevation, as well as of flexible floors, which are customary in historical constructions, no single mode can be identified as dominating the response and pushover analysis proves to be largely inaccurate. In addition, pushover analysis neglects the vertical component of ground motion.

To overcome these limitations, it is mandatory to resort to nonlinear dynamic analysis. The model for nonlinear dynamic analysis should account for a number of phenomena occurring in masonry: the progressive development of cracks, the energy dissipation in hysteresis loops and the decay of strength/stiffness under repeated cycles. In addition, masonry piers can fail either in bending or shear, with different hysteretic and decay characteristics.

Among the various approaches proposed, macroelement formulations [1,2,3] allow to satisfactorily describe the main global aspects of masonry structural response in a simplified fashion, representing a good compromise between accuracy and computational burden.

This study presents a macroelement for the nonlinear dynamic analysis of masonry buildings, according to the equivalent frame model $[1,2]$. This, formulated in a force-based framework, consists of a central linear elastic element, two flexural hinges at the ends and a shear link arranged in series, accounting for the hysteresis under cyclic loads. The flexural hinges and the shear link are modelled according to the Bouc-Wen (hereinafter referred to as BW) $[4,5]$ and the Bouc-Wen-Baber-Noori (hereinafter referred to as BWBN) [6] models, respectively. These are characterized by: a) a smooth transition between the elastic and the inelastic ranges, accounting for the progressive development of cracks; $b$ ) a small set of parameters able to reproduce hysteresis loops with different shapes. In particular, the bending hinges, showing low dissipation and negligible decay, are modelled by means of the BW model, whereas the shear link, with high dissipation and decay, by means of the BWBN model.

The macroelement and the solution algorithm for the element state determination have been implemented in the FEAP code [7], and numerical simulations of experimental tests on simple piers under axial force and shear are presented.

\section{CYCLIC CONSTITUTIVE LAWS FOR THE FLEXURAL AND SHEAR HINGES}

Bouc-Wen model has been used to describe hysteretic behaviour [8] in many mechanical and structural fields, including beam members [9,10], concrete walls [11], seismic isolation devices [12], wood joints [13], magneto-rheological fluid dampers [14,15], to cite some.

Its high versatility and the ability of reproducing a wide range of different hysteresis shapes, with a limited set of parameters, have prompted its introduction in the equivalent frame element formulation to simulate the behaviour of masonry panels.

According to this model, the restoring force is expressed as a linear combination of the elastic force, $P^{e l}$, and the history-dependent hysteretic term, $P^{h}$ :

$$
P=P^{e l}+P^{h}=a K u(t) v_{y}+(1-a) K z(t) v_{y}
$$

where $K$ is the tangent, initial stiffness, $a$ is the ratio between pre- and post yielding stiffness, $v_{\mathrm{y}}$ is the apparent yield displacement and $u$ has the meaning of ductility [16]. The evolution of the hysteretic variable $z(t)$ is ruled by the following differential equation:

$$
\dot{z}(t)=\left\{A-[\beta \operatorname{sign}(z(t) \dot{u}(t))+\gamma]|z(t)|^{n}\right\} \dot{u}(t)
$$


Equation (2) contains four non-dimensional parameters, $A, \beta, \gamma, n$, which govern the shape and the size of the hysteretic loop, $n$ affecting the abruptness of transition between pre- and post-yield response. The roles of these parameters and their influence on the cycle shape have been widely studied [16,17].

Based on Equation (1), the BW model can be represented by two springs, an elastic one and a hysteretic one, arranged in parallel, as reported in Figure 1.

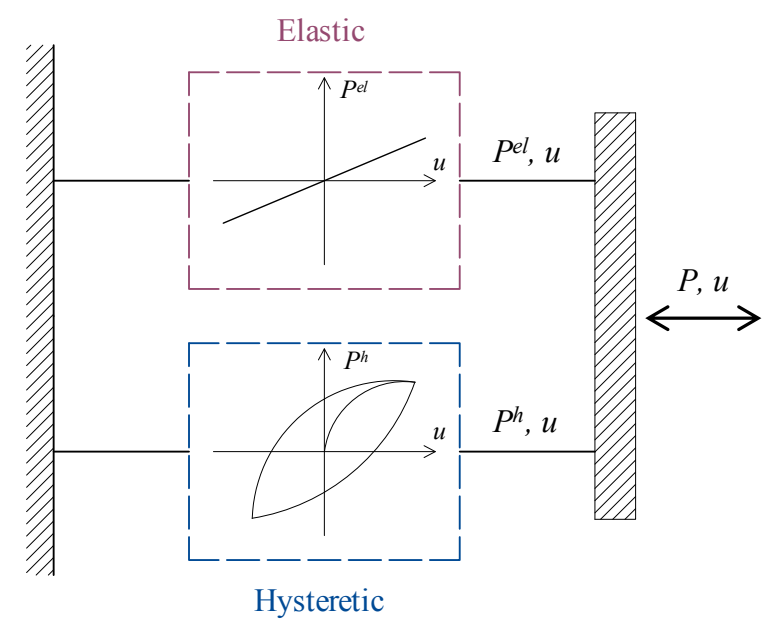

Figure 1: Two springs BW model representation.

Despite being extremely versatile, the BW hysteretic response does not take into account the effects of strength and stiffness degradation. This makes it suitable for the simulation of the masonry wall flexural response, which is typically characterized by a reduced amount of dissipation and small degradation effects. Conversely, BW model cannot reproduce the masonry shear mechanisms, which include relevant degradation effects.

Hence, to simulate the shear behaviour of masonry panels the BWBN model is introduced. This is an extension of the previous BW model, but is able to account for the effects of strength and stiffness degradation, by means of a set of energy-dependent parameters.

The differential equation, which rules the evolution of the hysteretic variable $z(t)$ according to the BWBN model, is:

$$
\dot{z}(t)=\frac{\left\{A(\varepsilon)-v(\varepsilon)[\beta \operatorname{sign}(z(t) \dot{u}(t))+\gamma]|z(t)|^{n}\right\} \dot{u}(t)}{\eta(\varepsilon)}
$$

where:

$$
\begin{aligned}
& \eta(\varepsilon)=\eta_{0}+\delta_{\eta} \varepsilon(t) \\
& v(\varepsilon)=v_{0}+\delta_{v} \varepsilon(t) \\
& A(\varepsilon)=A_{0}-\delta_{A} \varepsilon(t)
\end{aligned}
$$

with:

$$
\eta_{0}=v_{0}=A_{0}=1
$$

The energy related to the hysteretic spring is given by the following expression: 


$$
\varepsilon(t)=(1-a) K v_{y}^{2} \int_{u(0)}^{u(t)} z(t) d u=(1-a) K v_{y}^{2} \int_{0}^{t} z(t) \dot{u}(t) d t
$$

The introduced BW and BWBN formulations are used to model the constitutive response of the flexural and shear hinges, respectively. Thus, the displacement and force components in Equation (1), $v_{\mathrm{y}} u(t)$ and $P(t)$ are substituted by the kinematic and static variables governing the response of the mentioned hinges.

In the following, when referred to the flexural hinges, Equations (1) and (2) are applied to evaluate the couples $M_{1}$ and $M_{2}$ as function of the corresponding rotations $q_{1 b}$ and $q_{2 b}$, at hinges located at node $i$ and $j$, respectively. Similarly, Equations (1) and (3) are used to compute the response of the shear hinge, that is the shear force $T$ as function of the shear deformation $s$.

\section{FORCE-BASED BEAM FORMULATION}

The adopted beam formulation stands on the force-based approach, more efficient and accurate than the classical displacement-based models [18]. The beam is composed by the series arrangement of four sub-elements, a 2-node central elastic Timoshenko beam, two nonlinear flexural hinges located at the end nodes of the central element and a nonlinear shear link $[1,2]$. According to the equilibrated formulation, a basic reference system is introduced removing the rigid body motions from the six-component vector containing the beam nodal displacements. Thus, the resulting nodal kinematic parameters are collected in the vector $\mathbf{q}=\left[\begin{array}{l}q_{1} q_{2} q_{3}\end{array}\right]^{\mathrm{T}}, q_{1}$ and $q_{2}$ being the rotations at nodes $i$ and $j$, respectively, and $q_{3}$ the axial displacement. The workconjugated force vector $\mathbf{Q}=\left[\begin{array}{ll}Q_{1} & Q_{2} Q_{3}\end{array}\right]^{\mathrm{T}}$ collects the nodal force components, that is the two nodal couples $Q_{1}$ and $Q_{2}$ applied at nodes $i$ and $j$, respectively, and the axial force $Q_{3}$. Figure 2 shows the displacement (bottom) and force (top) nodal components in the (a) global and (b) basic reference system, respectively.

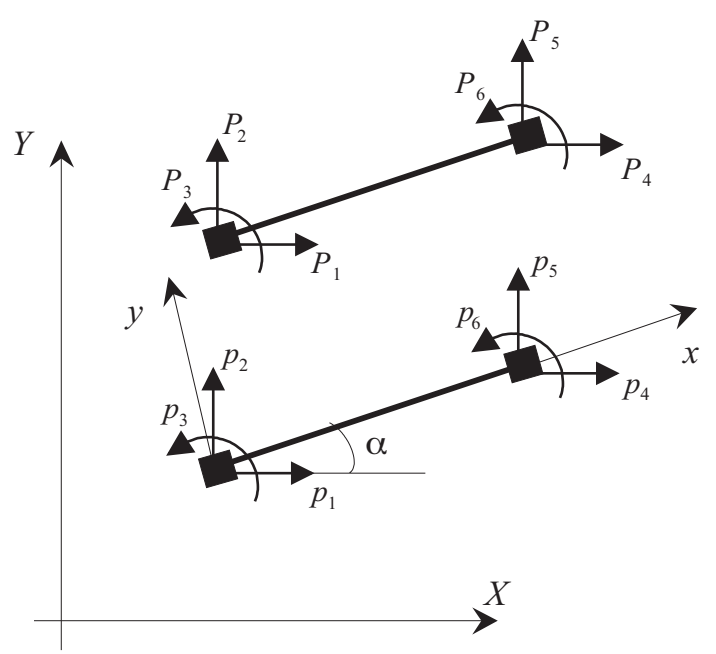

(a)

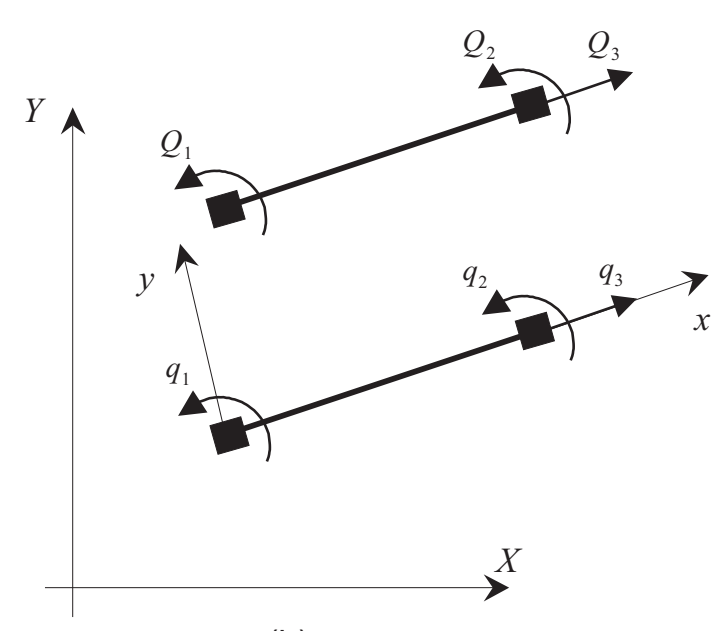

(b)

Figure 2: Beam finite element. Nodal forces (top) and displacement (bottom) in the (a) global and (b) basic reference system.

The following relation between the increments of the displacement and force vectors, $\dot{\mathbf{q}}$ and $\dot{\mathbf{Q}}$, holds:

$$
\dot{\mathbf{q}}=\mathbf{F} \dot{\mathbf{Q}}
$$


where the tangent flexibility matrix is derived by composing the elastic flexibility matrix of the central element and the tangent flexibility coefficients of the flexural and shear hinges. As a consequence of the series arrangement, it results:

$$
\mathbf{F}=\left[\begin{array}{ccc}
\frac{L}{3 E I}+\frac{1}{L G A^{*}}+F_{1 b}+\frac{F_{S}}{L^{2}} & -\frac{L}{6 E I}+\frac{1}{L G A^{*}}+\frac{F_{S}}{L^{2}} & 0 \\
-\frac{L}{6 E I}+\frac{1}{L G A^{*}}+\frac{F_{S}}{L^{2}} & \frac{L}{3 E I}+\frac{1}{L G A^{*}}+F_{2 b}+\frac{F_{S}}{L^{2}} & 0 \\
0 & 0 & \frac{L}{E A}
\end{array}\right]
$$

with $L$ being the element length, $E A, E I$ and $G A^{*}$ the axial, flexural and shear stiffness of the elastic beam element, $F_{1 b}, F_{2 b}$ and $F_{\mathrm{s}}$ the tangent flexibility coefficients of the flexural and shear hinges, respectively. To introduce the beam formulation in the global solution procedure based on the displacement method, matrix $\mathbf{F}$ is inverted to compute the element stiffness matrix $\mathbf{K}$ and the element nodal force vector $\mathbf{Q}$ has to be evaluated.

\subsection{Solution algorithm}

A step-by-step method for the time integration of the global equilibrium equations and a standard iterative Newton-Raphson algorithm are adopted. The beam element formulation is implemented in the FE analysis program FEAP [7], used to perform the numerical analyses. The global assembling procedure requires, at the element level, the computation of the element stiffness matrix and the structural reaction force vector. The adopted force-based approach involves an element state determination procedure more complex than the classical displacementbased formulation [18]. The main steps of this procedure are contained in Table 1.

$$
\begin{gathered}
\text { Element state determination } \\
\Delta \mathbf{q}^{k} \\
\Delta \mathbf{Q}^{k}=\left(\mathbf{F}^{k-1}\right)^{-1} \Delta \mathbf{q}^{k} \\
\Delta q_{1 b}^{k}=F_{1 b}^{k-1} \Delta Q_{1}^{k} ; \Delta q_{2 b}^{k}=F_{2 b}^{k-1} \Delta Q_{2}^{k} \\
\Delta s^{k}=F_{s}^{k-1}\left(\Delta Q_{1}^{k}+\Delta Q_{2}^{k}\right) / L \\
M_{1}^{k}, M_{2}^{k}, T^{k} ; \quad F_{1 b}^{k}, F_{2 b}^{k}, F_{s}^{k} \\
\mathbf{F}^{k} \\
r_{1 b}^{k}=F_{1 b}^{k}\left(Q_{1}^{k-1}+\Delta Q_{1}^{k}-M_{1}^{k}\right) \\
r_{2 b}^{k}=F_{2 b}^{k}\left(Q_{2}^{k-1}+\Delta Q_{2}^{k}-M_{2}^{k}\right) \\
r_{s}^{k}=\frac{F_{s}^{k}}{L}\left(\frac{Q_{1}^{k}+Q_{2}^{k}}{L}+\frac{\Delta Q_{1}^{k}+\Delta Q_{2}^{k}}{L}-T^{k}\right) \\
\mathbf{r}^{k}=\left[r_{1 b}^{k}+r_{s}^{k} r_{2 b}^{k}+r_{s}^{k} \quad 0\right]^{T} \\
\mathbf{Q}^{k}=\mathbf{Q}^{k-1}+\Delta \mathbf{Q}^{k}-\left(\mathbf{F}^{k}\right)^{-1} \mathbf{r}^{k}
\end{gathered}
$$

Table 1: Element state determination. 
After evaluating the increment of the element forces $\Delta \mathbf{Q}^{k}$, at the current Newton-Raphson iteration $k$, on the basis of the increment of the element displacements $\Delta \mathbf{q}^{k}$ and the element flexibility matrix at the previous iteration $\mathbf{F}^{k-1}$, the current increment of the hinge rotations, $\Delta q_{1 b}^{k}$ and $\Delta q_{2 b}^{k}$ and shear deformation $\Delta s^{k}$ are evaluated. Then, the flexural hinge rotations and the shear hinge deformation are updated and the constitutive responses are computed, giving the current hinge flexibilities, $F_{1 b}^{k}, F_{2 b}^{k}$ and $F_{s}^{k}$, as well as the moments and shear, $M_{1}^{k}, M_{2}^{k}, T^{k}$.

Hence, the current flexibility matrix of the overall element, $\mathbf{F}^{k}$, is updated and, finally, by its inversion, the new element stiffness $\mathbf{K}^{k}$ is computed. To evaluate the element reaction forces $\mathbf{Q}^{k}$, the kinematic residuals at the hinges, $r_{1 b}^{k}, r_{2 b}^{k}$ and $r_{s}^{k}$, are first determined, on the basis of the difference between the equilibrated forces and the values obtained by the constitutive laws. Such hinge residuals are transformed into the element kinematic residual, $\mathbf{r}^{k}$, by summing the contributions of the flexural and shear hinges at each node. By pre-multiplying $\mathbf{r}^{k}$ by the inverse of the element flexibility $\mathbf{F}^{k}$, a residual on the element reaction forces is calculated, used to compute the updated $\mathbf{Q}^{k}$. Both the updated stiffness matrix $\mathbf{K}^{k}=\left(\mathbf{F}^{k}\right)^{-1}$ and the nodal force vector $\mathbf{Q}^{k}$ are passed to the global code FEAP for the assembling and solution procedures. The element kinematic residual, $\mathbf{r}^{k}$, tends to vanish as the Newton-Raphson global iterations go on.

\section{ELEMENT VALIDATION}

Two masonry panels tested at the Joint Research Centre of Ispra under cyclic lateral displacement of increasing amplitude, applied quasi-statically to the top side are analysed [19]. These are characterized by a different $H / b$ ratio: a slender one $(b=1 \mathrm{~m}, H=2 \mathrm{~m})$ and a squat one ( $b=1 \mathrm{~m}, H=1.35 \mathrm{~m})$. The thickness $t$ is equal to $0.25 \mathrm{~m}$ for both panels. They are subjected to a constant vertical distributed load, whose resultant is $150 \mathrm{kN}$.

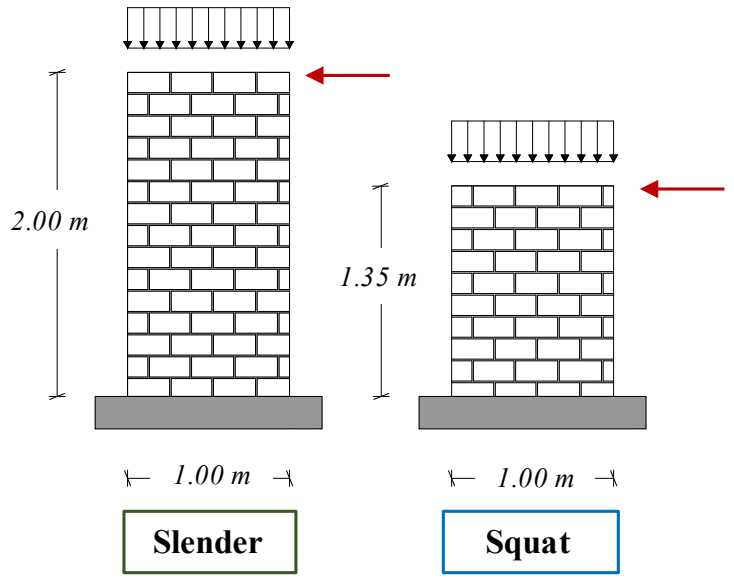

Figure 3: Ispra panels. Geometry and boundary conditions.

During the test, the top and bottom end sections were kept parallel.

The mechanical parameters have been deduced in [20] from experimental tests on components and small assemblages. The elastic parameters also adopted in the present study are $E=1.7$ $10^{6} \mathrm{kN} / \mathrm{m}^{2}$ and $G=0.310^{6} \mathrm{kN} / \mathrm{m}^{2}$. The parameters adopted for the BW and BWBN models are reported in Tables 2 and 3 .

First, the validation of the element is done by simulating the nonlinear behaviour of the panels, when subjected to an incremental static loading. A single FE is used to model the panel. The beam element is assumed as clamped at the base, with additional restraint at the top node affecting the rotation; therefore, the element is in double bending condition. 


\begin{tabular}{cccccc}
\hline$\beta$ & $\gamma$ & $n$ & $a$ & $K[\mathrm{kN} / \mathrm{mm}]$ & $v_{y}[\mathrm{~mm}]$ \\
\hline 0.2 & 0.8 & 1 & 0.005 & 36.94 & 1.8 \\
\hline
\end{tabular}

Table 2: Parameters of the BW model for slender panel.

\begin{tabular}{ccccccccc}
\hline$\beta$ & $\gamma$ & $n$ & $a$ & $K[\mathrm{kN} / \mathrm{mm}]$ & $v_{y}[\mathrm{~mm}]$ & $\delta \eta$ & $\delta v$ & $\delta_{A}$ \\
\hline 0.5 & 0.5 & 1 & 0.000 & 89.57 & 1.2 & 0.002 & 0.0008 & 0 \\
\hline
\end{tabular}

Table 3: Parameters of the BWBN model for squat panel.

Figure 5 shows the pushover response curves for the (a) slender and (b) squat panel, respectively, in terms of base shear versus top displacement, obtained by using the proposed macroelement formulation (square symbols), compared with the experimental envelope curves (triangle symbols) and with the results presented in [2] (circle symbols), where a rigid-perfectly plastic behaviour has been assumed for the hinges.
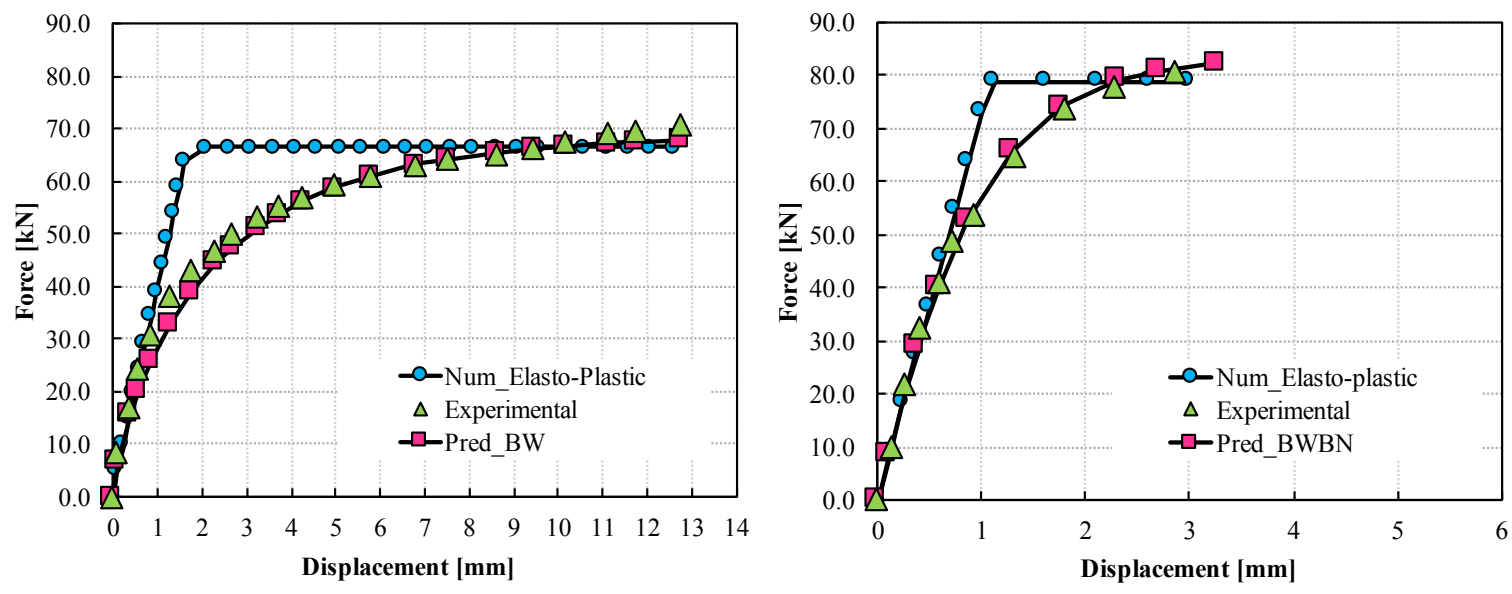

Figure 5: Pushover response curves for slender a) and squat b) panel.

A very good agreement between the experimental envelope curves and those obtained with the proposed macroelement is noted.

Then, the nonlinear cyclic behaviour of the panels has been investigated and another validation has been done comparing the experimental cyclic response curves with the results of the proposed model simulation. The mechanical parameters are those contained in Tables 2 and 3; in this case, the energy-dependent coefficients are relevant to reproduce the strength and stiffness degradation. Both the experimental response and the proposed model predictions are shown in Figure 6 (a) and (b) for the slender and the squat panel respectively, where a satisfactory match between the experimental and the numerical results emerges. 

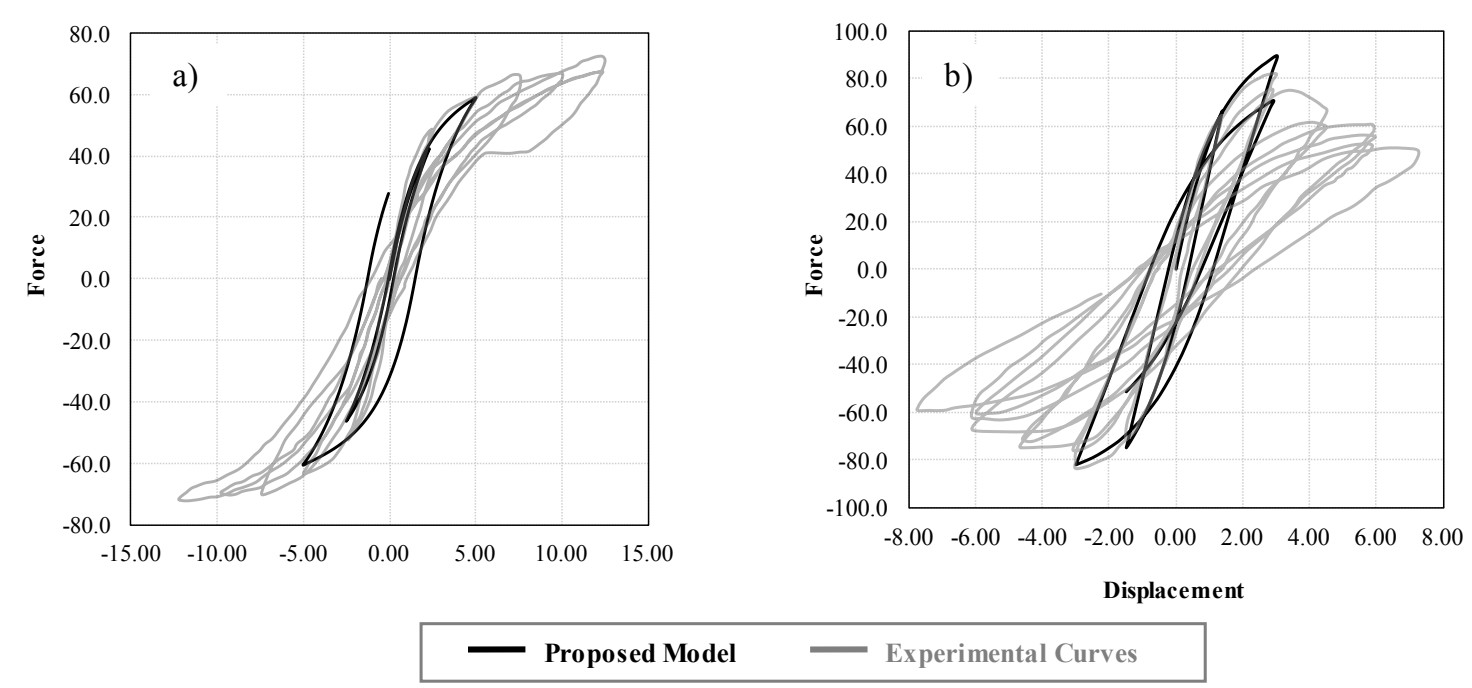

Figure 6: Experimental response of Ispra panels and proposed model predictions for a) slender and b) squat panel

\section{CONCLUDING REMARKS}

- A force-based macrolement has been presented, where the nonlinear masonry behaviour has been modelled by introducing two flexural hinges at the end nodes and a shear link.

- The adoption of the BW and BWBN models for the constitutive response of the hinges is a novelty introduced by this study. This has allowed to reproduce the hysteresis and the stiffness-strength decay, typically characterizing the main nonlinear mechanisms of masonry walls.

- A beam FE and a proper solution algorithm have been developed and introduced in the FEAP code, used to perform the numerical applications.

- The presented macroelement formulation has been validated by analysing two experimentally tested simple piers, where the typical flexural and shear mechanisms emerge. The comparison between the numerical and experimental results show that the element is accurate in reproducing the experimental hysteresis loops and the strength/stiffness decay of masonry. At the same time, its computation burden is much lower than that of micro- or multiscale models, making it suitable to analyse medium to large constructions.

\section{REFERENCES}

[1] D. Addessi, D. Liberatore, R. Masiani, Force-Based Beam Finite Element (FE) for the Pushover Analysis of Masonry Buildings. International Journal of Architectural Heritage: Conservation, Analysis, and Restoration, 9, 231-243, 2015.

[2] D. Liberatore, D. Addessi, Strength domains and return algorithm for the lumped plasticity equivalent frame model of masonry structures. Engineering Structures, 91, 167-181, 2015.

[3] D. Addessi, A. Mastrandrea, E. Sacco, An equilibrated macro-element for nonlinear analysis of masonry structures. Engineering Structures, 70, 82-93, 2014.

[4] R. Bouc, Forced vibration of mechanical systems with hysteresis. 4th Conference on Nonlinear Oscillation, Prague, Czechoslovakia, 1967. 
[5] Y.K. Wen, Method for random vibration of hysteretic systems. Journal of the Engineering Mechanics Division, 102(2), 249-263, 1976.

[6] T.T. Baber, M.N. Noori, Random vibration of degrading, pinching systems. Journal of the Engineering Mechanics Division, 111(8), 1010-1026, 1985.

[7] R.L. Taylor, FEAP - a finite element analysis program, version 8.3. Department of Civil and Environmental Engineering, University of California at Berkeley, Berkeley, 2011.

[8] M. Ismail, F. Ikhouane, J. Rodellar, The hysteresis Bouc-Wen model, a survey. Archives of Computational Methods in Engineering, 16(2), 161-188, 2009.

[9] M. Saatcioglu, G. Ozcebe, Response of reinforced concrete columns to simulated seismic loading. ACI Structural Journal, 86(1), 3-12, 1989.

[10] S.P. Triantafyllou, V.K. Koumousis, Small and large displacement dynamic analysis of frame structures based on hysteretic beam elements. Journal of the Engineering Mechanics Division, 138(1), 36-49, 2011.

[11] I.D. Lefas, M.D. Kotsovos, Strength and deformation characteristics of reinforced concrete walls under load reversals. ACI Structural Journal, 87(6), 716-726, 1990.

[12] T. Sireteanu, M. Giuclea, A.M. Mitu, Identification of an extended Bouc-Wen model with application to seismic protection through hysteretic devices. Computational Mechanics, 45(5), 431-441, 2010.

[13] G.C. Foliente, Hysteresis modeling of wood joints and structural systems. Journal of Structural Engineering, 121(6), 1013-1022, 1995.

[14] N.M. Kwok, Q.P. Ha, T.H. Nguyen, J. Li, B. Samali, A novel hysteretic model for magnetorheological fluid dampers and parameter identification using particle swarm optimization. Sensors and Actuators, 132, 441-451, 2006.

[15] S. Talatahari, A. Kaveh, N.M. Rahbari, Parameter identification of Bouc-Wen model for MR fluid dampers using adaptive charged system search optimization. Journal of Mechanical Science and Technology, 26(8), 2523-2534, 2012.

[16] F. Ikhouane, J. Rodellar, J.E. Hurtado, Analytical Characterization of Hysteresis Loops Described by the Bouc-Wen Model. Mechanics of Advanced Materials and Structures, 13(6), 463-472, 2006.

[17] F. Ikhouane, J.E. Hurtado, J. Rodellar, Variation of the hysteresis loop with the BoucWen model parameters. Nonlinear Dynamics, 48(4), 361-380, 2007.

[18] D. Addessi, V. Ciampi, A regularized force-based beam element with a damage-plastic section constitutive law. International Journal for Numerical Methods in Engineering, 70, 610-629, 2007.

[19] A. Anthoine, G. Magonette, G. Magenes, Shear-compression testing and analysis of brick masonry walls. 10th European conference on earthquake engineering, Balkema, Rotterdam, The Netherlands, 1995.

[20] G. Magenes, G.M. Calvi, In-plane seismic response of brick masonry walls. Earthquake Eng Struct Dyn, 26, 1091-1112, 1997. 